\title{
The fixed point property of Orlicz sequence spaces equipped with the $p$-Amemiya norm
}

\author{
Xin $\mathrm{He}^{1,2^{*}}$, Yunan Cui and Henryk Hudzik
}

\section{"Correspondence:}

hexin8323@163.com

'Department of Mathematics,

Harbin Institute of Technology,

Harbin, 150001, P.R. China

${ }^{2}$ Department of Mathematics,

Harbin Normal University, Harbin,

150025, P.R. China

Full list of author information is

available at the end of the article

\begin{abstract}
In this paper, the Opial modulus and the weakly convergent sequence coefficient of Orlicz space $I_{\phi, p}$ endowed with the $p$-Amemiya norm are calculated, the criteria for the uniform Opial property as well as for weakly uniform normal structure of $I_{\phi, p}$ are presented. It is shown that the Orlicz sequence space equipped with the $p$-Amemiya norm has the fixed point property if and only if it is reflexive.
\end{abstract}

MSC: Primary 47H10; secondary 46E30; 46B20

Keywords: Orlicz sequence space; Opial property; weakly convergent sequence coefficient; weakly uniform normal structure; $p$-Amemiya norm

\section{Introduction and preliminaries}

The aim of this paper is to present criteria for some important geometric properties related to the metric fixed point theory in Orlicz sequence spaces.

The Opial property originates from the fixed point theorem proved by Opial in [1]. The uniform Opial property with respect to the weak topology was defined in [2] and Opial modulus was introduced in [3]. It is well known that the Opial property and normal structure of a Banach space $X$ play an important role in metric fixed point theory for nonexpansive mappings, as well as in the theory of differential and integral equations (see [1, 4-6]). The Opial property also plays an important role in the study of weak convergence of iterates, random products of nonexpansive mappings and the asymptotic behavior of nonlinear semigroups [1,7-9]. Moreover, it can be introduced to the open unit ball of a complex Hilbert space, equipped with the hyperbolic metric, where it is useful in proving the existence of fixed points of holomorphic self-mappings of $X$ [8].

The coefficient WCS $(X)$ was introduced by Bynum [10], who established their relations with normal structure and calculated the value of $\mathrm{WCS}\left(l^{p}\right)$. A reflexive Banach space $X$ with $\operatorname{WCS}(X)>1$ has normal structure and consequently it has the weakly fixed point property. This is probably one of the Banach space constants which has been most widely studied, although with considerable confusion because there exist many equivalent definitions. Several different formulae for $\operatorname{WCS}(X)$ were found (see [4]), also see the work by Sims and Smyth [11].

The notion of $p$-Amemiya norm was introduced by Cui and Hudzik in [12], where they showed that the $p$-Amemiya norm $\|\cdot\|_{\Phi, p}$ is equivalent to the Orlicz norm $\|\cdot\|_{\Phi}^{\circ}$ as well as to the Luxemburg norm $\|\cdot\|_{\Phi}$. They also illustrated the description of extreme points and strongly extreme points in Orlicz spaces equipped with the $p$-Amemiya norm 
$[12,13]$. In 2012, they presented the criteria for non-squareness, uniform non-squareness, and locally uniform non-squareness of these spaces [14]. Chen and Cui (see [15, 16]) gave the criteria for complex extreme points and complex strict convexity in Orlicz function spaces equipped with the $p$-Amemiya norm, and for complex mid-point locally uniform rotundity and complex rotundity of Orlicz sequence spaces equipped with the $p$-Amemiya norm.

The rest of the paper is organized as follows. In the first section, some basic notions, terminology and original results are reviewed, which will be used throughout the paper. In Section 2, the Opial modulus of Orlicz space $l_{\phi, p}$ endowed with the $p$-Amemiya norm is calculated, and the criteria for the uniform Opial property of $l_{\phi, p}$ are presented. The weakly convergent sequence coefficient is calculated in Section 3. Finally, the necessary and sufficient condition for fixed point properties to exist in $l_{\phi, p}$ are given.

Let $X$ be a Banach space. We denote by $B(X)$ the unit ball of $X$, by $S(X)$ the unit sphere of $X$. Now we recall some notions from fixed point theory.

A mapping $T: C \rightarrow X$ defined on a subset $C$ of a Banach space $X$ is said to be nonexpansive if $\|T x-T y\| \leq\|x-y\|$ for all $x, y \in C$.

We say that a Banach space $X$ has the fixed point property if for every weakly compact convex subset $C \subset X$ and for every nonexpansive $T: C \rightarrow C, T$ has a fixed point of $C$.

It is known that $L^{1}[0,1]$ does not have the fixed point property.

For any map $\Phi: \mathbb{R} \rightarrow[0, \infty]$, define

$$
a_{\Phi}=\max \{u \geq 0: \Phi(u)=0\}, \quad b_{\Phi}=\max \{u \geq 0: \Phi(u)<\infty\} .
$$

A map $\Phi$ is said to be an Orlicz function if $\Phi(0)=0, \Phi$ is not identically equal to zero, it is even and convex on the interval $\left(-b_{\Phi}, b_{\Phi}\right)$ and left-continuous at $b_{\Phi}$.

For every Orlicz function $\Phi$, we define its complementary function $\Psi: \mathbb{R} \rightarrow[0, \infty]$ by the formula

$$
\Psi(v)=\sup \{u|v|-\Phi(u): u \geq 0\}
$$

And the convex modular by $I_{\Phi}(x)=\sum_{i=1}^{\infty} \Phi(x(i))$ for any $x=(x(i))$.

Definition 1.1 [17-19] The Orlicz sequence space is defined as the set

$$
l_{\Phi}=\left\{x=(x(i)): I_{\Phi}(\lambda x)<\infty \text { for some } \lambda>0\right\} .
$$

The Luxemburg norm and the Orlicz norm are expressed as

$$
\|x\|_{\Phi}=\inf \left\{\lambda>0: I_{\Phi}\left(\frac{x}{\lambda}\right) \leq 1\right\}
$$

and

$$
\|x\|_{\Phi}^{\circ}=\inf _{k>0} \frac{1}{k}\left(1+I_{\Phi}(k x)\right)
$$

respectively. The Orlicz space equipped with the Luxemburg norm and the Orlicz norm is denoted by $l_{\Phi}$ and $l_{\Phi}^{\circ}$, respectively. 
For any $1 \leq p \leq \infty$ and $u \geq 0$, define

$$
s_{p}(u)= \begin{cases}\left(1+u^{p}\right)^{\frac{1}{p}} & \text { for } 1 \leq p<\infty \\ \max \{1, u\} & \text { for } p=\infty\end{cases}
$$

and define $s_{\Phi, p}(x)=s_{p} \circ I_{\Phi}(x)$ for all $1 \leq p \leq \infty$. Note that the functions $s_{p}$ and $s_{\Phi, p}$ are convex. Moreover, the function $s_{p}$ is increasing on $\mathbb{R}_{+}$for $1 \leq p<\infty$, but the function $s_{\infty}$ is increasing on the interval $[1, \infty)$ only.

Definition $1.2[12,13]$ Let $1 \leq p \leq \infty$. For any $x=(x(i))$, define the $p$-Amemiya norm by the formula

$$
\|x\|_{\Phi, p}=\inf _{k>0} \frac{1}{k} s_{\Phi, p}(k x)
$$

The Orlicz space equipped with the $p$-Amemiya norm will be denoted by $l_{\Phi, p}$.

It is known that $\|x\|_{\Phi, 1}=\|x\|_{\Phi}^{\circ}$ and $\|x\|_{\Phi, \infty}=\|x\|_{\Phi}$. If $1<p<\infty$ and $x \neq 0$,

$$
\frac{1}{2}\|x\|_{\Phi}^{\circ} \leq\|x\|_{\Phi} \leq\|x\|_{\Phi, p} \leq 2^{\frac{1}{p}}\|x\|_{\Phi}<2^{\frac{1}{p}}\|x\|_{\Phi}^{\circ}
$$

(see [12]).

Let $p_{+}$be the right-hand side derivative of $\Phi$ on $\left[0, b_{\Phi}\right)$ and put $p_{+}\left(b_{\Phi}\right)=\lim _{u \rightarrow b_{\Phi}^{-}} p_{+}(u)$. Define the function $\alpha_{p}: l_{\Phi, p} \rightarrow[-1, \infty]$ by

$$
\alpha_{p}(x)= \begin{cases}I_{\Phi}^{p-1}(x) I_{\Psi}\left(p_{+}(|x|)\right)-1, & 1 \leq p<\infty \\ -1, & p=\infty, I_{\Phi}(x) \leq 1 \\ I_{\Psi}\left(p_{+}(|x|)\right), & p=\infty, I_{\Phi}(x)>1\end{cases}
$$

and the functions $k_{p}^{*}: l_{\Phi, p} \rightarrow[0, \infty), k_{p}^{* *}: l_{\Phi, p} \rightarrow[0, \infty)$ by

$$
\begin{aligned}
& k_{p}^{*}(x)=\inf \left\{k \geq 0: \alpha_{p}(k x) \geq 0\right\} \quad(\text { with } \inf \phi=\infty), \\
& k_{p}^{* *}(x)=\inf \left\{k \geq 0: \alpha_{p}(k x) \leq 0\right\} .
\end{aligned}
$$

It is obvious that $k_{p}^{*}(x) \leq k_{p}^{* *}(x)$ for every $1 \leq p \leq \infty$ and $x \in l_{\Phi, p}$.

Set $K_{p}(x)=\left\{0<k<\infty: k_{p}^{*}(x) \leq k \leq k_{p}^{* *}(x)\right\}$.

Definition 1.3 [17] We say that an Orlicz function $\Phi$ satisfies the $\Delta_{2}(0)$-condition $(\Phi \in$ $\Delta_{2}(0)$, for short) if there exist constants $K \geq 2$ and $u_{0}>0$ such that

$$
\Phi(2 u) \leq K \Phi(u) \quad \text { for every }|u| \leq u_{0}
$$

For more details about Orlicz spaces, we refer to $[13,14,18,19]$.

Lemma 1.1 [14] Let $1 \leq p \leq \infty$. Then $I_{\Phi}(x) \leq\|x\|_{\Phi, p}$ for all $x \in L_{\Phi, p}$ with $\|x\|_{\Phi, p} \leq 1$.

Lemma 1.2 [17] If the Orlicz function $\Phi$ vanishes only at zero and $\Phi \in \Delta_{2}(0)$, then the norm convergence and the modular convergence are equivalent. 
Lemma 1.3 [12] For every $1 \leq p \leq \infty$ and each $x \in l_{\Phi, p} \backslash\{0\}$, the following conditions hold.

1. If $k_{p}^{*}(x)=k_{p}^{* *}(x)=\infty, K_{p}(x)=\phi$, then

$$
\|x\|_{\Phi, p}=\lim _{k \rightarrow \infty} \frac{1}{k}\left(1+I_{\Phi}^{p}(k x)\right)^{\frac{1}{p}} .
$$

2. If $k_{p}^{*}(x)<k_{p}^{* *}(x)=\infty$, then the $p$-Amemiya norm $\|x\|_{\Phi, p}$ is attained at every $k \in\left[k_{p}^{*}(x), \infty\right)$.

3. If $k_{P}^{* *}(x)<\infty$, then the $p$-Amemiya norm $\|x\|_{\Phi, p}$ is attained at every $k \in\left[k_{p}^{*}(x), k_{p}^{* *}(x)\right]$.

Lemma 1.4 If the Orlicz function $\Phi$ vanishes only at zero, then $l_{\Phi, p}$ is order continuous if and only if $\Phi \in \Delta_{2}(0)$.

Lemma 1.5 Assume $\Phi \in \Delta_{2}(0), 1 \leq p<\infty$. Then, for any $L>0$ and $\varepsilon>0$, there exists $\delta>0$ such that

$$
I_{\Phi}(x) \leq L, \quad I_{\Phi}(y) \leq \delta \quad \Rightarrow \quad\left|I_{\Phi}^{p}(x+y)-I_{\Phi}^{p}(x)\right|<\varepsilon \quad\left(x, y \in l_{\Phi, p}\right) .
$$

Proof Let

$$
h=\sup \left\{I_{\Phi}(2 x+2 y): I_{\Phi}(x) \leq L, I_{\Phi}(y) \leq 1\right\} .
$$

Then $L<h<\infty$, since $\Phi \in \Delta_{2}(0)$. Without loss of generality, we assume $L>1$ and $\varepsilon<1$. Set $\beta=\frac{\varepsilon}{h^{p}}$. Since the modular convergence implies the norm convergence, so we can find $\delta>0$ such that $I_{\Phi}(y) \leq \delta$ implies $\|y\|_{\Phi, p} \leq \min \left\{\frac{\beta}{2}, \frac{\varepsilon^{\frac{1}{p}} \beta^{1-\frac{1}{p}}}{2}\right\}$. Thus, applying the convexity of $\Phi$ and Lemma 1.1, we have

$$
\begin{aligned}
I_{\Phi}^{p}(x+y) & =I_{\Phi}^{p}\left((1-\beta) x+\beta\left(x+\beta^{-1} y\right)\right) \\
& \leq(1-\beta) I_{\Phi}^{p}(x)+\beta I_{\Phi}^{p}\left(x+\beta^{-1} y\right) \\
& \leq(1-\beta) I_{\Phi}^{p}(x)+\frac{\beta}{2} I_{\Phi}^{p}(2 x)+\frac{\beta}{2} I_{\Phi}^{p}\left(2 \beta^{-1} y\right) \\
& \leq(1-\beta) I_{\Phi}^{p}(x)+\frac{\beta}{2} I_{\Phi}^{p}(2 x)+\frac{\beta}{2}\left\|2 \beta^{-1} y\right\|_{\Phi, p}^{p} \\
& \leq I_{\Phi}^{p}(x)+\frac{\beta h^{p}}{2}+\frac{2^{p-1}}{\beta^{p-1}}\|y\|_{\Phi, p}^{p} \\
& \leq I_{\Phi}^{p}(x)+\varepsilon .
\end{aligned}
$$

Replacing $x, y$ by $x+y,-y$, respectively, in the above inequalities, we have

$$
I_{\Phi}^{p}(x) \leq I_{\Phi}^{p}(x+y)+\varepsilon
$$

Lemma 1.6 [20] $l_{\Phi, p}$ is reflexive if and only if $\Phi \in \Delta_{2}(0)$ and $\Psi \in \Delta_{2}(0)$.

Lemma 1.7 Let $1 \leq p<\infty$. $l_{\Phi, p}$ has a subspace isomorphic to $l^{1}$ if and only if $A>0$, where $A=\lim _{u \rightarrow 0} \frac{\Phi(u)}{u}$. 
Proof Since the function $\frac{\Phi(u)}{u}$ is nondecreasing, $\lim _{u \rightarrow 0} \frac{\Phi(u)}{u}$ exists.

If $A>0$, by the continuity of $\frac{\Phi(u)}{u}$, there exists $A_{1}>0$ such that

$$
A|u| \leq \Phi(u) \leq A_{1}|u| \quad\left(|u| \in\left[0, \Phi^{-1}(1)\right]\right) .
$$

Then $\Phi \in \Delta_{2}(0)$.

For any $x \in l_{\Phi, p}$, we have $I_{\Phi}\left(\frac{x}{\|x\|_{\Phi}}\right)=1$, then $\frac{|x(i)|}{\|x\|_{\Phi}} \in\left[0, \Phi^{-1}(1)\right](i=1,2, \ldots)$, therefore,

$$
A \frac{|x(i)|}{\|x\|_{\Phi}} \leq \Phi\left(\frac{|x(i)|}{\|x\|_{\Phi}}\right) \leq A_{1} \frac{|x(i)|}{\|x\|_{\Phi}}
$$

we have

$$
A \sum_{i=1}^{\infty} \frac{|x(i)|}{\|x\|_{\Phi}} \leq \sum_{i=1}^{\infty} \Phi\left(\frac{|x(i)|}{\|x\|_{\Phi}}\right) \leq A_{1} \sum_{i=1}^{\infty} \frac{|x(i)|}{\|x\|_{\Phi}},
$$

then $A\|x\|_{l^{1}} \leq\|x\|_{\Phi} \leq A_{1}\|x\|_{l^{1}}$. This yields that the $l^{1}$ norm and the Luxemburg norm are equivalent. Since the $p$-Amemiya norm and the Luxemburg norm are equivalent, $l_{\Phi, p}$ has a subspace isomorphic to $l^{1}$.

Therefore, if $A>0$, the space $l_{\Phi, p}$ has the Schur property, and it has the Opial property trivially because there is no weakly null sequence $\left(x_{n}\right)$ in $S\left(l_{\Phi}\right)$. The case when $A>0$ is not interesting if we consider the Opial modulus and weakly convergent sequence coefficient. For this reason we will assume that $A=0$ in the following whenever the Opial modulus and the weakly convergent sequence coefficient are considered.

\section{Opial modulus for Orlicz sequence spaces}

In this section we present some results on the Opial modulus. The obtained results extend the existing ones, which were presented by a number of papers studying the geometry of Orlicz spaces endowed with the Luxemburg norm and the Orlicz norm, respectively. A formula for calculating the Opial modulus in Orlicz and Musielak-Orlicz spaces equipped with the Luxemburg or the Orlicz norm is found in [21-26].

Definition 2.1 [1] We say that a Banach space $X$ has the Opial property if for any weakly null sequence $\left(x_{n}\right)$ in $X$ and any $x \in X \backslash\{0\}$ there holds

$$
\liminf _{n \rightarrow \infty}\left\|x_{n}\right\|<\liminf _{n \rightarrow \infty}\left\|x+x_{n}\right\|
$$

Opial proved in [1] that $l^{p}(1<p<\infty)$ has this property, but $L^{p}[0,2 \pi]$ does not have it if $p \in(1, \infty), p \neq 2$.

Definition 2.2 [6] We say that $X$ has the uniform Opial property if for any $\varepsilon>0$ there exists $r>0$ such that for any $x \in X$ with $\|x\| \geq \varepsilon$ and any weakly null sequence $\left(x_{n}\right)$ in the unit sphere $S(X)$ of $X$ there holds

$$
1+r \leq \liminf _{n \rightarrow \infty}\left\|x_{n}+x\right\|
$$


It is obvious that the uniform Opial property implies the Opial property.

Definition 2.3 [2] The Opial modulus of $X$ is denoted by $\delta_{o}$ and it is defined for $\varepsilon \in(0 ; 1]$ by the formula

$$
\delta_{o}(\varepsilon)=\inf \left\{\liminf _{n \rightarrow \infty}\left\|x_{n}+x\right\|:\left(x_{n}\right) \subset S(X), x_{n} \rightarrow 0 \text { weakly, }\|x\|=\varepsilon\right\} .
$$

It is easy to see that $X$ has the uniform Opial property if and only if $\delta_{o}(\varepsilon)>1$ for any $\varepsilon \in(0,1]$.

Theorem 2.1 If $\Phi$ is an Orlicz function, $1 \leq p<\infty, a_{\Phi}>0$, then $l_{\Phi, p}$ does not have the Opial property.

Proof Divide $\mathbb{N}$ into a sequence $\left(N_{n}\right)$ of pairwise disjoint and infinite subsets of $\mathbb{N}$ such that $\inf _{n} N_{n} \rightarrow \infty$ as $n \rightarrow \infty$ and define

$$
x_{n}=\sum_{i \in N_{n+1}} a_{\Phi} e_{i}, \quad x=\sum_{i \in N_{1}} a_{\Phi} e_{i}
$$

Then the sequence $\left(x_{n}\right)$ is weakly convergent to zero. For any $k>1$, we have

$$
I_{\Phi}\left(k x_{n}\right)=I_{\Phi}(k x)=I_{\Phi}\left(k\left(x_{n}+x\right)\right)=\infty
$$

and for any $k \in(0,1]$,

$$
\frac{1}{k}\left(1+I_{\Phi}^{p}\left(k x_{n}\right)\right)^{\frac{1}{p}}=\frac{1}{k}\left(1+I_{\Phi}^{p}(k x)\right)^{\frac{1}{p}}=\frac{1}{k}\left(1+I_{\Phi}^{p}\left(k\left(x_{n}+x\right)\right)\right)^{\frac{1}{p}}=\frac{1}{k} .
$$

So $\left\|x_{n}\right\|_{\Phi, p}=\|x\|_{\Phi, p}=\left\|x_{n}+x\right\|_{\Phi, p}=1$. Therefore, $l_{\Phi, p}$ does not have the Opial property.

In the following we may assume that $a_{\Phi}=0$.

Theorem 2.2 Let $\Phi$ be an Orlicz function satisfying $\Delta_{2}(0), 1 \leq p<\infty$. Then for any $\varepsilon \in$ $(0,1]$, we have

$$
\begin{gathered}
\delta_{o}(\varepsilon)=\inf \left\{c_{x y k}>0: I_{\Phi}^{p}\left(\frac{k x}{c_{x y k}}\right)+I_{\Phi}^{p}\left(\frac{k y}{c_{x y k}}\right)=k^{p}-1,\right. \\
\left.k>1,\|x\|_{\Phi, p}=1,\|y\|_{\Phi, p}=\varepsilon, x, y \in l_{\Phi, p}\right\} .
\end{gathered}
$$

Proof Set $d(\varepsilon)=\inf \left\{c_{x y k}>0: I_{\Phi}^{p}\left(\frac{k x}{c_{x y k}}\right)+I_{\Phi}^{p}\left(\frac{k y}{c_{x y k}}\right)=k^{p}-1, k>1,\|x\|_{\Phi, p}=1,\|y\|_{\Phi, p}=\varepsilon, x, y \in\right.$ $\left.l_{\Phi, p}\right\}$.

(1) For fixed $k>1$, consider the function

$$
F(c)=I_{\Phi}^{p}\left(\frac{k x}{c}\right)+I_{\Phi}^{p}\left(\frac{k y}{c}\right)
$$

We have $F$ is continuous on $\mathbb{R}_{+}$. Since

$$
1=\|x\|_{\Phi, p} \leq \frac{1}{k}\left(1+I_{\Phi}^{p}(k x)\right)^{\frac{1}{p}}
$$


we have $I_{\Phi}^{p}(k x) \geq k^{p}-1$ for any $k>0$, thus

$$
F(1)=I_{\Phi}^{p}(k x)+I_{\Phi}^{p}(k y)>k^{p}-1
$$

Moreover, $\lim _{c \rightarrow+\infty} F(c)=0$, then there exists unique $c_{x y k} \in(1,+\infty)$ such that

$$
F\left(c_{x y k}\right)=I_{\Phi}^{p}\left(\frac{k x}{c_{x y k}}\right)+I_{\Phi}^{p}\left(\frac{k y}{c_{x y k}}\right)=k^{p}-1,
$$

which shows that the function $d(\varepsilon)$ is well defined.

(2) Now, we will show that $\delta_{o}(\varepsilon) \leq d(\varepsilon)$ for any $\varepsilon \in(0,1]$. For any $\theta>0$, there exist $x_{0}, y_{0} \in$ $X$ with $\left\|x_{0}\right\|=1,\left\|y_{0}\right\|=\varepsilon$ and $k_{0}>1$ such that $d(\varepsilon)+\theta>c_{x_{0} y_{0} k_{0}}$. Put

$$
\begin{aligned}
& x=\left(y_{0}(1), 0, y_{0}(2), 0, y_{0}(3), 0, y_{0}(4), 0, \ldots\right), \\
& x_{n}=\sum_{i=0}^{\infty} x_{0}(i+1) e_{2 n+2^{n} i} \quad(n=1,2, \ldots) .
\end{aligned}
$$

Then $\left\|x_{n}\right\|_{\Phi, p}=\left\|x_{0}\right\|_{\Phi, p}=1,\|x\|_{\Phi, p}=\left\|y_{0}\right\|_{\Phi, p}=\varepsilon$, and $x_{n}(i) \rightarrow 0$ as $n \rightarrow \infty$ for any $i \in \mathbb{N}$. Since $\sum_{i=1}^{\infty} \Phi(x(i))<\infty$, there exists $i_{0} \in \mathbb{N}$ such that $\sum_{i=i_{0}+1}^{\infty} \Phi(x(i))<\theta$.

For fixed $i_{0}$, due to $\lim _{u \rightarrow 0} \frac{\Phi(u)}{u}=0$, we have

$$
\begin{aligned}
\lim _{l \rightarrow 0} \frac{1}{l} \sum_{i=1}^{i_{0}} \Phi(l x(i)) & =\lim _{l \rightarrow 0} \sum_{i=1}^{i_{0}} \frac{\Phi(l x(i))}{l|x(i)|}|x(i)| \\
& =\sum_{i=1}^{i_{0}} \lim _{l \rightarrow 0} \frac{\Phi(l x(i))}{l|x(i)|}|x(i)|=0 .
\end{aligned}
$$

So, for any $n \in \mathbb{N}$ and $l \in(0,1)$ which is small enough, we get

$$
\begin{aligned}
\frac{I_{\Phi}\left(l x_{n}\right)}{l} & =\frac{I_{\Phi}(l x)}{l}=\frac{1}{l}\left(\sum_{i=1}^{i_{0}} \Phi(l x(i))+\sum_{i=i_{0}+1}^{\infty} \Phi(l x(i))\right) \\
& \leq \frac{1}{l} \sum_{i=1}^{i_{0}} \Phi(l x(i))+\sum_{i=i_{0}+1}^{\infty} \Phi(x(i))<2 \theta .
\end{aligned}
$$

Then $\lim _{l \rightarrow 0} \frac{I_{\Phi}\left(l x_{n}\right)}{l}=0$.

Take any $f \in l_{\Psi, q}$, there exists $\lambda>0$ satisfying $I_{\Psi}(\lambda f)<\infty$. Since

$$
\inf \left(\operatorname{supp} x_{n}\right) \rightarrow \infty \text { as } n \rightarrow \infty \text {, }
$$

as well as the Young inequality, we have

$$
\left|f\left(x_{n}\right)\right|=\left|\sum_{i=1}^{\infty} x_{n}(i) f(i)\right| \leq \frac{1}{l \lambda}\left(I_{\Phi}\left(l x_{n}\right)+I_{\Psi}\left(\lambda f \chi_{\text {supp } x_{n}}\right)\right) \rightarrow 0
$$

$($ as $n \rightarrow \infty)$. Then we have proved that $x_{n} \rightarrow 0$ weakly. 
Since $x$ and $x_{n}$ have disjoint supports, we get

$$
\begin{aligned}
\frac{\left\|x_{n}+x\right\|_{\Phi, p}}{d(\varepsilon)+\theta} & \leq \frac{1}{k_{0}}\left(1+I_{\Phi}^{p}\left(\frac{k_{0}\left(x_{n}+x\right)}{d(\varepsilon)+\theta}\right)\right)^{\frac{1}{p}} \\
& =\frac{1}{k_{0}}\left(1+I_{\Phi}^{p}\left(\frac{k_{0} x_{n}}{d(\varepsilon)+\theta}\right)+I_{\Phi}^{p}\left(\frac{k_{0} x}{d(\varepsilon)+\theta}\right)\right)^{\frac{1}{p}} \\
& \leq \frac{1}{k_{0}}\left(1+I_{\Phi}^{p}\left(\frac{k_{0} x_{n}}{c_{x_{0} y_{0} k_{0}}}\right)+I_{\Phi}^{p}\left(\frac{k_{0} x}{c_{x_{0} y_{0} k_{0}}}\right)\right)^{\frac{1}{p}}=1,
\end{aligned}
$$

hence $\left\|x_{n}+x\right\|_{\Phi, p} \leq d(\varepsilon)+\theta$. By the arbitrariness of $\theta>0$, we have $\delta_{o}(\varepsilon) \leq d(\varepsilon)$.

(3) Assume that $\delta_{o}(\varepsilon)<d(\varepsilon)$ for some $\varepsilon \in(0,1]$. Then there exists $\varepsilon_{0}>0$ such that $\delta_{o}(\varepsilon) \leq$ $d(\varepsilon)-2 \varepsilon_{0}$, so there exist $x \in l_{\Phi, p}$ and $\left(x_{n}\right)$ in $S\left(l_{\Phi, p}\right)$ such that $\|x\|_{\Phi, p}=\varepsilon, x_{n} \rightarrow 0$ weakly and

$$
\lim _{n \rightarrow \infty}\left\|x_{n}+x\right\|_{\Phi, p}<d(\varepsilon)-2 \varepsilon_{0}
$$

For fixed $\frac{\varepsilon_{0}}{4}$, there exists $i_{0}$ such that

$$
\left\|\sum_{i=i_{0}+1}^{\infty} x(i) e_{i}\right\|_{\Phi, p}<\frac{\varepsilon_{0}}{4}, \quad\left\|\sum_{i=1}^{i_{0}} x_{n}(i) e_{i}\right\|_{\Phi, p}<\frac{\varepsilon_{0}}{4}
$$

for $n \in \mathbb{N}$ large enough. Set

$$
\left\|\sum_{i=1}^{i_{0}} x(i) e_{i}\right\|_{\Phi, p}=a, \quad\left\|\sum_{i=i_{0}+1}^{\infty} x_{n}(i) e_{i}\right\|_{\Phi, p}=b_{n} .
$$

And define

$$
\begin{aligned}
& y=\left(\frac{\varepsilon x(1)}{a}, \frac{\varepsilon x(2)}{a}, \ldots, \frac{\varepsilon x\left(i_{0}\right)}{a}, 0,0, \ldots\right), \\
& y_{n}=\left(0,0, \ldots 0, \frac{x_{n}\left(i_{0}+1\right)}{b_{n}}, \frac{x_{n}\left(i_{0}+2\right)}{b_{n}}, \ldots, \frac{x_{n}\left(i_{0}+m\right)}{b_{n}}, \ldots\right),
\end{aligned}
$$

then $\left\|y_{n}\right\|_{\Phi, p}=1$ (for all $\left.n \in \mathbb{N}\right),\|y\|_{\Phi, p}=\varepsilon, y_{n} \stackrel{w}{\rightarrow} 0$. For $n$ large enough, we have

$$
\begin{aligned}
\left\|y_{n}+y\right\|_{\Phi, p} \leq & \left\|\sum_{i=i_{0}+1}^{\infty} \frac{x_{n}(i)}{b_{n}} e_{i}-x_{n}\right\|_{\Phi, p}+\left\|\sum_{i=1}^{i_{0}} \frac{\varepsilon}{a} x(i) e_{i}-x\right\|_{\Phi, p}+\left\|x_{n}+x\right\|_{\Phi, p} \\
\leq & \left\|\sum_{i=1}^{i_{0}} x_{n}(i) e_{i}\right\|_{\Phi, p}+\left|1-b_{n}\right|+\left\|\sum_{i=i_{0}+1}^{\infty} x(i) e_{i}\right\|_{\Phi, p} \\
& +|\varepsilon-a|+d(\varepsilon)-2 \varepsilon_{0} \\
\leq & 2\left\|\sum_{i=1}^{i_{0}} x_{n}(i) e_{i}\right\|_{\Phi, p}+2\left\|\sum_{i=i_{0}+1}^{\infty} x(i) e_{i}\right\|_{\Phi, p}+d(\varepsilon)-2 \varepsilon_{0} \\
< & d(\varepsilon)-\varepsilon_{0} .
\end{aligned}
$$


Case 1. $A=\left\{n \in \mathbb{N}: K_{\Phi}\left(\frac{y_{n}+y}{d(\varepsilon)}\right) \neq \phi\right\}$ is infinite. For any $n \in A$, we take $k_{n} \in K_{\Phi}\left(\frac{y_{n}+y}{d(\varepsilon)}\right)$. Then

$$
\begin{aligned}
1-\frac{\varepsilon_{0}}{d(\varepsilon)} & \geq \frac{\left\|y_{n}+y\right\|_{\Phi, p}}{d(\varepsilon)} \\
& =\frac{1}{k_{n}}\left(1+I_{\Phi}^{p}\left(\frac{k_{n}\left(y_{n}+y\right)}{d(\varepsilon)}\right)\right)^{\frac{1}{p}} \\
& =\frac{1}{k_{n}}\left(1+I_{\Phi}^{p}\left(\frac{k_{n} y_{n}}{d(\varepsilon)}\right)+I_{\Phi}^{p}\left(\frac{k_{n} y}{d(\varepsilon)}\right)\right)^{\frac{1}{p}} \\
& \geq \frac{1}{k_{n}}\left(1+I_{\Phi}^{p}\left(\frac{k_{n} y_{n}}{c_{y_{n} y k_{n}}}\right)+I_{\Phi}^{p}\left(\frac{k_{n} y}{c_{y_{n} y k_{n}}}\right)\right)^{\frac{1}{p}}=1 .
\end{aligned}
$$

This is a contradiction.

Case 2. $B=\left\{n \in \mathbb{N}: K_{\Phi}\left(\frac{y_{n}+y}{d(\varepsilon)}\right)=\phi\right\}$ is infinite. For any $n \in B$,

$$
\begin{aligned}
1-\frac{\varepsilon_{0}}{d(\varepsilon)} & \geq \frac{\left\|y_{n}+y\right\|_{\Phi, p}}{d(\varepsilon)} \\
& =\lim _{l \rightarrow \infty} \frac{1}{l}\left(1+I_{\Phi}^{p}\left(\frac{l\left(y_{n}+y\right)}{d(\varepsilon)}\right)\right)^{\frac{1}{p}} \\
& =\lim _{l \rightarrow \infty} \frac{1}{l}\left(1+I_{\Phi}^{p}\left(\frac{l y_{n}}{d(\varepsilon)}\right)+I_{\Phi}^{p}\left(\frac{l y}{d(\varepsilon)}\right)\right)^{\frac{1}{p}} \\
& \geq \lim _{l \rightarrow \infty} \frac{1}{l}\left(1+I_{\Phi}^{p}\left(\frac{l y_{n}}{c_{y_{n} y l}}\right)+I_{\Phi}^{p}\left(\frac{l y}{c_{y_{n} y l}}\right)\right)^{\frac{1}{p}}=1,
\end{aligned}
$$

this is also a contradiction. The two cases have shown that $\delta_{o}(\varepsilon)=d(\varepsilon)$.

Remark The main result presented in this paper generalizes the existing result to the $p$-Amemiya norm. In the case that $p=1$, the situation degrades to the case of the classical Orlicz norm.

If $\Phi \in \Delta_{2}(0)$, then

$$
\begin{gathered}
\delta_{o}(\varepsilon)=\inf \left\{c_{x y k}>0: I_{\Phi}\left(\frac{k x}{c_{x y k}}\right)+I_{\Phi}\left(\frac{k y}{c_{x y k}}\right)=k-1,\right. \\
\left.k>1,\|x\|_{\Phi}^{\circ}=1,\|y\|_{\Phi}^{\circ}=\varepsilon, x, y \in l_{\Phi}^{\circ}\right\} .
\end{gathered}
$$

In the following we will consider the uniform Opial property for the Orlicz sequence equipped with the $p$-Amemiya norm.

Lemma 2.3 [25] Let $X$ be a Köthe sequence space with the semi-Fatou property and without order continuity of the norm. Then $X$ does not have the uniform Opial property. In fact, we even have that for any $\varepsilon \in(0,1), \delta_{o}(\varepsilon)=1$.

Theorem 2.4 Let $1 \leq p<\infty, l_{\Phi, p}$ has the uniform Opial property if and only if $\Phi \in \Delta_{2}(0)$.

Proof If $\Phi \in \Delta_{2}(0)$ and $l_{\Phi, p}$ does not have the uniform Opial property, then there exists some $\varepsilon_{0} \in(0,1]$ such that $\delta_{o}\left(\varepsilon_{0}\right)=1$. Therefore, for any sequence $\left(c_{n}\right)$ such that $c_{n} \searrow 1$, 
there are two sequences $\left(x_{n}\right)$ and $\left(y_{n}\right)$ in $l_{\Phi, p}$ with $\left\|x_{n}\right\|=1,\left\|y_{n}\right\|=\varepsilon_{0}$ and $k_{n}>1$ such that

$$
I_{\Phi}^{p}\left(\frac{k_{n} x_{n}}{c_{n}}\right)+I_{\Phi}^{p}\left(\frac{k_{n} y_{n}}{c_{n}}\right)=k_{n}^{p}-1 .
$$

Since

$$
\begin{aligned}
& \frac{1}{c_{n}}=\frac{\left\|x_{n}\right\|_{\Phi, p}}{c_{n}} \leq \frac{1}{k_{n}}\left(1+I_{\Phi}^{p}\left(\frac{k_{n} x_{n}}{c_{n}}\right)\right)^{\frac{1}{p}}, \\
& \frac{\varepsilon_{0}}{c_{n}}=\frac{\left\|y_{n}\right\|_{\Phi, p}}{c_{n}} \leq \frac{1}{k_{n}}\left(1+I_{\Phi}^{p}\left(\frac{k_{n} y_{n}}{c_{n}}\right)\right)^{\frac{1}{p}},
\end{aligned}
$$

then

$$
I_{\Phi}^{p}\left(\frac{k_{n} x_{n}}{c_{n}}\right) \geq\left(\frac{k_{n}}{c_{n}}\right)^{p}-1, \quad I_{\Phi}^{p}\left(\frac{k_{n} y_{n}}{c_{n}}\right) \geq\left(\frac{\varepsilon_{0} k_{n}}{c_{n}}\right)^{p}-1 .
$$

Thus we obtain $\left(\frac{k_{n}}{c_{n}}\right)^{p}-1+\left(\frac{\varepsilon_{0} k_{n}}{c_{n}}\right)^{p}-1 \leq k_{n}^{p}-1$, then $k_{n} \leq \frac{c_{n}^{p}}{1+\varepsilon_{0}^{p}}$, which means that the sequence $\left(k_{n}\right)$ is bounded. Then

$$
I_{\Phi}\left(\frac{k_{n} y_{n}}{c_{n}}\right)=k_{n}^{p}-1-I_{\Phi}^{p}\left(\frac{k_{n} x_{n}}{c_{n}}\right) \leq k_{n}^{p}-1-\left(\frac{k_{n}}{c_{n}}\right)^{p}+1 \rightarrow 0 .
$$

But $\left\|\frac{k_{n} y_{n}}{c_{n}}\right\|_{\Phi, p}=\frac{\varepsilon_{0} k_{n}}{c_{n}}>\frac{\varepsilon_{0}}{c_{n}} \rightarrow \varepsilon_{0}$, according to Lemma 1.2 and $\Phi \in \Delta_{2}(0)$, this is a contradiction.

If $\Phi \notin \Delta_{2}(0)$, then $l_{\Phi, p}$ does not have the order continuity property, by Lemma 2.3 , the proof is finished.

\section{Weakly convergent sequence coefficient for Orlicz sequence spaces}

In this section, our main aim is to calculate the weakly convergent sequence coefficient for an Orlicz sequence space and further discuss the fixed point property of this space.

Let $X$ denote a reflexive infinite dimensional Banach space, without Schur property automatically. For each sequence $\left(x_{n}\right)$ in $X$, we define the asymptotic diameter and asymptotic radius respectively by

$$
\begin{aligned}
& \operatorname{dian}_{a}\left(x_{n}\right)=\limsup _{k \rightarrow \infty}\left\{\left\|x_{n}-x_{m}\right\|: n, m \geq k\right\}, \\
& r_{a}\left(x_{n}\right)=\inf \left\{\limsup _{n \rightarrow \infty}\left\|x_{n}-y\right\|: y \in \overline{\operatorname{conv}}\left(x_{n}\right)\right\} .
\end{aligned}
$$

The weakly convergent sequence coefficient concerned with normal structure is an important geometric parameter. It was introduced by Bynum [10] as follows.

$\mathrm{WCS}(X)$ is the supremum of the set of all numbers $M$ with the property that for each weakly convergent sequence $\left(x_{n}\right)$ with asymptotic diameter $A$, there is some $y$ in the closed convex hull of the sequence such that

$$
M \limsup \left\|x_{n}-y\right\| \leq A \text {. }
$$

$n$

A sequence $\left(x_{n}\right)$ in $X$ is said to be asymptotic equidistant if

$$
\operatorname{dian}_{a}\left(x_{n}\right)=\liminf _{n \rightarrow \infty}\left\{\left\|x_{i}-x_{j}\right\|: i \neq j, i, j \geq n\right\} .
$$


In this paper, we use the following equivalent definition of $\operatorname{WCS}(X)$. This definition was introduced in [27], where it was proved that

$\operatorname{WCS}(X)=\inf \left\{\operatorname{dian}_{a}\left(x_{n}\right):\left(x_{n}\right)\right.$ is an asymptotic equidistant sequence in

$$
\left.(X) \text { and } x_{n} \rightarrow 0 \text { weakly }\right\} \text {. }
$$

It is obvious that $1 \leq \mathrm{WCS}(X) \leq 2$ (see [10]). A Banach space $X$ is said to have weakly uniform normal structure provided $\operatorname{WCS}(X)>1$. See [11] for further information about this coefficient.

For $p \geq 1, \operatorname{WSC}\left(l^{p}\right)=2^{\frac{1}{p}}$ and $\operatorname{WCS}\left(L^{p}(\Omega)\right)=\min \left\{2^{\frac{1}{p}}, 2^{1-\frac{1}{p}}\right\}$. A formula for calculating the weakly convergent sequences of reflexive Orlicz and Musielak-Orlicz sequence spaces equipped with the Luxemburg or Amemiya norm is found in [21, 23], respectively.

Theorem 3.1 If $\Phi \in \Delta_{2}(0), 1 \leq p<\infty$, then

$$
\operatorname{WCS}\left(l_{\Phi, p}\right)=\inf \left\{\inf _{k>1}\left\{c_{x k}: I_{\Phi}^{p}\left(\frac{k x}{c_{x k}}\right)=\frac{k^{p}-1}{2}\right\}: x \in S\left(l_{\Phi, p}\right)\right\} .
$$

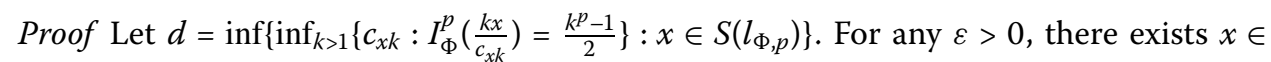
$S\left(l_{\Phi, p}\right)$ such that

$$
\inf \left\{\inf _{k>1}\left\{c_{x k}: I_{\Phi}^{p}\left(\frac{k x}{c_{x k}}\right)=\frac{k^{p}-1}{2}\right\}: x \in S\left(l_{\Phi, p}\right)\right\}<d+\varepsilon .
$$

Then there exist $k>1$ and $c_{x k}<d+\varepsilon$ such that $I_{\Phi}^{p}\left(\frac{k x}{c_{x k}}\right)=\frac{k^{p}-1}{2}$. Define

$$
\begin{aligned}
& x_{1}=(x(1), 0, x(2), 0, x(3), 0, x(4), 0, x(5), 0, x(6), 0, \ldots), \\
& x_{2}=(0, x(1), 0,0,0, x(2), 0,0,0,0,0,0,0, x(3), 0,0,0, \ldots), \\
& x_{3}=(0,0,0, x(1), 0,0,0,0,0,0,0, x(2), 0,0,0,0,0,0,0, \ldots),
\end{aligned}
$$

where $\left(x_{n}\right)$ have pairwise disjoint supports. Then $\left\|x_{n}\right\|_{\Phi, p}=\|x\|_{\Phi, p}=1(n \in \mathbb{N})$ for all $n \neq m$ and all $k>1$. According to the same method as in the proof of Theorem 2.2, we have $x_{n} \rightarrow 0$ weakly,

$$
\begin{aligned}
\left\|\frac{x_{n}-x_{m}}{d+\varepsilon}\right\|_{\Phi, p} & \leq \frac{1}{k}\left(1+I_{\Phi}^{p}\left(k \frac{x_{n}-x_{m}}{d-\varepsilon}\right)\right)^{\frac{1}{p}} \\
& =\frac{1}{k}\left(1+2 I_{\Phi}^{p}\left(\frac{k x}{d+\varepsilon}\right)\right)^{\frac{1}{p}} \\
& <\frac{1}{k}\left(1+2 I_{\Phi}^{p}\left(\frac{k x}{c_{x k}}\right)\right)^{\frac{1}{p}} \\
& =\frac{1}{k}\left(1+k^{p}-1\right)^{\frac{1}{p}}=1 .
\end{aligned}
$$

Then $\left\|x^{n}-x^{m}\right\|_{\Phi, p} \leq d+\varepsilon$, so $\operatorname{dian}_{a}\left(x_{n}\right) \leq d+\varepsilon$, since $\varepsilon$ is arbitrary, we have $\operatorname{WCS}\left(l_{\Phi, p}\right) \leq d$. 
He et al. Fixed Point Theory and Applications 2013, 2013:340

Page 12 of 18

On the other hand, let $\left\{x_{n}\right\}$ in $S\left(l_{\Phi, p}\right)$ be an arbitrary asymptotic equidistant sequence such that $x_{n} \rightarrow 0$ weakly.

Since $\Phi \in \Delta_{2}(0)$, then by Lemma 1.5 for $\varepsilon>0$, there exists $0<\delta<\varepsilon$ such that

$$
I_{\Phi}(x) \leq 1, \quad k \leq \frac{1}{\varepsilon} \quad \text { and } \quad I_{\Phi}(y) \leq \delta \quad \Rightarrow \quad\left|I_{\Phi}^{p}\left(\frac{k(x+y)}{d}\right)-I_{\Phi}^{p}\left(\frac{k x}{d}\right)\right|<\varepsilon .
$$

Let $n_{1}=1$ and pick $m_{1}$ such that $\sum_{j>m_{1}} \Phi\left(\frac{x_{n_{1}}(j)}{\varepsilon d}\right)<\delta$ and choose $n_{2}>n_{1}$ such that $\sum_{j \leq m_{1}} \Phi\left(\frac{x_{n_{2}}(j)}{\varepsilon d}\right)<\delta$. By $x_{n}(i) \rightarrow 0$ as $n \rightarrow \infty$ for $i=1,2, \ldots$, we can find $m_{2}$ with $m_{2}>m_{1}$ such that $\sum_{j>m_{2}} \Phi\left(\frac{x_{n_{2}}(j)}{\varepsilon d}\right)<\delta$. And so on, by induction, we find the sequence $\left\{n_{i}\right\}$ and $\left\{m_{i}\right\}$ of natural numbers with $n_{1}<n_{2}<\cdots, m_{1}<m_{2}<\cdots$ satisfying

$$
\sum_{j>m_{i}} \Phi\left(\frac{x_{n_{i}}(j)}{\varepsilon d}\right)<\delta, \quad \sum_{j \leq m_{i}} \Phi\left(\frac{x_{n_{i}}(j)}{\varepsilon d}\right)<\delta .
$$

Take $k_{i j} \in K_{\Phi}\left(\frac{x_{n_{i}}-x_{n_{j}}}{d}\right)$.

(1) If $k_{i j} \leq 1$, then $\left\|\frac{x_{n_{i}}-x_{n_{j}}}{d}\right\|_{\Phi, p} \geq \frac{1}{k_{i j}} \geq 1$, so $\left\|x_{n_{i}}-x_{n_{j}}\right\| \geq d$.

(2) If $1<k_{i j} \leq \frac{1}{\varepsilon}$, set $n<m$, then

$$
\begin{aligned}
& \left\|\frac{x_{n_{i}}-x_{n_{j}}}{d}\right\|_{\Phi, p}^{p} \\
& \quad \geq \frac{1}{k_{i j}^{p}}\left(1+\left(\sum_{l \leq m_{i}} \Phi\left(\frac{k_{i j} x_{n_{i}}(l)}{d}\right)\right)^{p}-\varepsilon+\left(\sum_{l>m_{i}} \Phi\left(\frac{k_{i j} x_{n_{i}}(l)}{d}\right)\right)^{p}-\varepsilon\right) \\
& \quad \geq \frac{1}{k_{i j}^{p}}\left(1+I_{\Phi}^{p}\left(\frac{k_{i j} x_{n_{i}}(l)}{d}\right)-\delta-\varepsilon+I_{\Phi}^{p}\left(\frac{k_{i j} x_{n_{i}}(l)}{d}\right)-\delta-\varepsilon\right) \\
& \geq \frac{1}{k_{i j}^{p}}\left(1+I_{\Phi}^{p}\left(\frac{k_{i j} x_{n_{i}}(l)}{c_{x_{n_{i}} k_{i j}}}\right)+I_{\Phi}^{p}\left(\frac{k_{i j} x_{n_{i}}(l)}{c_{x_{n_{i}} k_{i j}}}\right)-4 \varepsilon\right) \\
& \quad=\frac{1}{k_{i j}^{p}}\left(1+2 \cdot \frac{k_{i j}^{p}-1}{2}-4 \varepsilon\right)>1-4 \varepsilon .
\end{aligned}
$$

Therefore $\left\|x_{n_{i}}-x_{n_{j}}\right\|_{\Phi, p} \geq d$.

(3) If $k_{i j} \geq \frac{1}{\varepsilon}$, then

$$
\begin{aligned}
\left\|\frac{x_{n_{i}}-x_{n_{j}}}{d}\right\|_{\Phi, p}^{p} & >\frac{1}{k_{i j}^{p}} I_{\Phi}^{p}\left(k_{i j} \frac{x_{n_{i}}-x_{n_{j}}}{d}\right) \\
& \geq \varepsilon^{p} I_{\Phi}^{p}\left(\frac{x_{n_{i}}-x_{n_{j}}}{\varepsilon d}\right) \\
& \geq \varepsilon^{p}\left(I_{\Phi}^{p}\left(\frac{x_{n_{i}}}{\varepsilon d}\right)+I_{\Phi}^{p}\left(\frac{x_{n_{j}}}{\varepsilon d}\right)-4 \varepsilon\right) \\
& \geq \varepsilon^{p}\left(I_{\Phi}^{p}\left(\frac{x_{n_{i}}}{\varepsilon c_{x_{n_{i}} \frac{1}{\varepsilon}}}\right)+I_{\Phi}^{p}\left(\frac{x_{n_{j}}}{\varepsilon c_{x_{n_{j}} \frac{1}{\varepsilon}}}\right)-4 \varepsilon\right) \\
& =\varepsilon^{p}\left(\varepsilon^{-p}-1-4 \varepsilon\right)=1-\varepsilon^{p}-4 \varepsilon^{p+1},
\end{aligned}
$$


hence we get $\left\|x_{n_{i}}-x_{n_{j}}\right\|_{\Phi, p} \geq d$ again. Consequently $\operatorname{dian}_{a}\left(x_{n}\right) \geq d$. By the arbitrariness of $\left\{x_{n}\right\}$ in $S\left(l_{\Phi, p}\right)$, it follows that $\operatorname{WCS}\left(l_{\Phi, p}\right) \geq d$.

Theorem 3.2 Let $1 \leq p<\infty$ and $a_{\Phi}=0 . l_{\Phi, p}$ has weakly uniform normal structure if and only if $\Phi \in \Delta_{2}(0)$.

Proof Necessity. If $\Phi \in \Delta_{2}(0)$, by Theorem 3.1,

$$
\operatorname{WCS}\left(l_{\Phi, p}\right)=\inf \left\{\inf _{k>1}\left\{c_{x k}: I_{\Phi}^{p}\left(\frac{k x}{c_{x k}}\right)=\frac{k^{p}-1}{2}\right\}: x \in S\left(l_{\Phi, p}\right)\right\} .
$$

Assume $\operatorname{WCS}\left(l_{\Phi, p}\right)=1$, then for any $0<\varepsilon<1$ there exist $x \in S\left(l_{\Phi, p}\right)$ and $k>1$ satisfying $c_{x k}<1+\varepsilon$. By $\|x\|_{\Phi, p}=1$ and $\Phi \in \Delta_{2}(0)$, there exists $\delta>0$ such that $I_{\Phi}\left(\frac{x}{2}\right) \geq \delta$. Hence,

$$
\begin{aligned}
1 & =\frac{1}{k^{p}}\left(2 I_{\Phi}^{p}\left(\frac{k x}{c_{x k}}\right)+1\right) \\
& \geq \frac{1}{k^{p}}\left(1+I_{\Phi}^{p}\left(\frac{k x}{1+\varepsilon}\right)\right)+\frac{1}{k^{p}} I_{\Phi}^{p}\left(\frac{k x}{1+\varepsilon}\right) \\
& \geq\left\|\frac{x}{1+\varepsilon}\right\|_{\Phi, p}^{p}+I_{\Phi}^{p}\left(\frac{x}{1+\varepsilon}\right) \\
& \geq\left(\frac{1}{1+\varepsilon}\right)^{p}+I_{\Phi}^{p}\left(\frac{x}{2}\right) \\
& \geq\left(\frac{1}{1+\varepsilon}\right)^{p}+\delta^{p} \rightarrow 1+\delta^{p} \quad \text { as } \varepsilon \rightarrow 0 .
\end{aligned}
$$

This is a contradiction.

Sufficiency. If not, $\Phi \notin \Delta_{2}(0)$, then for any $\varepsilon>0$ there exists $0<u<\varepsilon$ such that

$$
\Phi((1+\varepsilon) u)>\frac{1}{\varepsilon} \Phi(u)
$$

We can find $m \in \mathbb{N}$ such that

$$
1-\Phi(2 \varepsilon)<m \Phi((1+\varepsilon) u) \leq 1
$$

Take $c>0$ satisfying $m \Phi((1+\varepsilon) u)+\Phi(c)=1$. Then $\Phi(c)<\Phi(2 \varepsilon)$. Set

$$
\begin{aligned}
& x_{1}=(c \overbrace{(1+\varepsilon) u, \ldots,(1+\varepsilon) u}^{m}, 0,0, \ldots), \\
& x_{2}=(\overbrace{0, \ldots, 0}^{m+1}, c, \overbrace{(1+\varepsilon) u, \ldots,(1+\varepsilon) u}^{m}, 0,0, \ldots), \\
& x_{3}=(\overbrace{0, \ldots, 0}^{2 m+2}, c, \overbrace{(1+\varepsilon) u, \ldots,(1+\varepsilon) u}^{m}, 0,0, \ldots),
\end{aligned}
$$


Then $\frac{x_{n}}{\left\|x_{n}\right\|_{\Phi, p}} \in S\left(l_{\Phi, p}\right)$ and $\left\{\frac{x_{n}}{\left\|x_{n}\right\|_{\Phi, p}}\right\}$ is an asymptotic equidistant sequence. And because $\lim \sup _{l \rightarrow 0} \frac{I_{\Phi}\left(l x_{n}\right)}{l}=0$, we have $x_{n} \rightarrow 0$ weakly. Finally, $\left\|x_{n}\right\|_{\Phi, p}=\left\|x_{1}\right\|_{\Phi, p} \geq\left\|x_{1}\right\|_{\Phi, \infty}=1$, then

$$
\begin{aligned}
& \left\|\frac{1}{1+\varepsilon}\left(\frac{x_{n}}{\left\|x_{n}\right\|}-\frac{x_{m}}{\left\|x_{m}\right\|}\right)\right\|_{\Phi, p}^{p} \\
& \quad \leq\left\|\frac{x_{n}-x_{m}}{1+\varepsilon}\right\|_{\Phi, p}^{p} \leq 1+I_{\Phi}^{p}\left(\frac{x_{n}-x_{m}}{1+\varepsilon}\right) \\
& \quad=1+\left(2 m \Phi(u)+2 \Phi\left(\frac{c}{1+\varepsilon}\right)\right)^{p} \\
& \quad \leq 1+(2 m \varepsilon \Phi((1+\varepsilon) u)+2 \Phi(c))^{p} \\
& \quad \leq 1+(2 \varepsilon+2 \Phi(2 \varepsilon))^{p}
\end{aligned}
$$

which implies that

$$
\operatorname{dian}_{a}\left(x_{n}\right) \leq(1+\varepsilon)\left(1+(2 \varepsilon+2 \Phi(2 \varepsilon))^{p}\right)
$$

so $\operatorname{WCS}\left(l_{\Phi, p}\right)=1$.

According to the above proof, we have the following.

Corollary 3.3 Let $1 \leq p<\infty, a_{\Phi}=0$ and $\Phi \notin \Delta_{2}(0)$, then $\operatorname{WCS}\left(l_{\Phi, p}\right)=1$.

Remark In the case that $p=1$, the situation degrades to the case of classical Orlicz norm.

1. If $\Phi \notin \Delta_{2}(0)$, then $\operatorname{WCS}\left(l_{\Phi}^{\circ}\right)=1$.

2. If $\Phi \in \Delta_{2}(0)$, then

$$
\operatorname{WCS}\left(l_{\Phi}^{\circ}\right)=\inf \left\{\inf _{k>1}\left\{c_{x k}: I_{\Phi}\left(\frac{k x}{c_{x k}}\right)=\frac{k-1}{2}\right\}: x \in S\left(l_{\Phi}^{\circ}\right)\right\} .
$$

\section{Corollary 3.4}

$$
\operatorname{WCS}\left(l^{p}\right)= \begin{cases}\frac{1}{2^{p}}, & 1 \leq p<\infty \\ 1, & p=\infty\end{cases}
$$

Next, we discuss the fixed point property of $l_{\Phi, p}$.

Theorem $3.5 \Phi \notin \Delta_{2}(0)$ and $a_{\Phi}=0,1 \leq p \leq \infty$, then $l_{\Phi, p}$ contains an asymptotically isometric copy of $c_{0}$.

Proof If $\Phi \notin \Delta_{2}(0)$, then there exist the sequence $\left\{u_{k}\right\} \downarrow 0$ and $\left\{n_{k}\right\} \subset \mathbb{N}$ such that

$$
\begin{aligned}
& \Phi\left(u_{k}\right) \leq \frac{1}{2^{k+\frac{1}{p}+1}}, \quad \frac{1}{2^{k+\frac{1}{p}+1}} \leq n_{k} \Phi\left(u_{k}\right) \leq \frac{1}{2^{k+\frac{1}{p}}} \\
& \Phi\left(\left(1+\frac{1}{k}\right) u_{k}\right)>2^{k+\frac{1}{p}+1} \Phi\left(u_{k}\right) \quad \text { for all } k \in \mathbb{N} .
\end{aligned}
$$


Set

$$
\begin{aligned}
& x_{1}=(\overbrace{u_{1}, \ldots, u_{1}}^{n_{1}}, 0,0, \ldots, 0, \ldots), \\
& x_{2}=(\overbrace{0, \ldots, 0}^{n_{1}}, \overbrace{u_{2}, \ldots, u_{2}}^{n_{2}}, 0,0, \ldots, 0, \ldots), \\
& x_{3}=(\overbrace{0, \ldots, 0}^{n_{1}+n_{2}}, \overbrace{u_{3}, \ldots, u_{3}}^{n_{3}}, 0,0, \ldots, 0, \ldots),
\end{aligned}
$$

Define $P: c_{0} \rightarrow l_{\Phi, p}$ by

$$
P t=\sum_{n=1}^{\infty} t_{n} x_{n}
$$

for $t=\left(t_{1}, t_{2}, \ldots\right) \in c_{0}$. It is obvious that $P$ is linear.

For any $\lambda>0$, since $t_{n} \rightarrow 0$, then there exists $j_{0} \in \mathbb{N}$ such that $\lambda\left|t_{i}\right|<1$ for all $i \geq j_{0}$, hence

$$
\begin{aligned}
I_{\Phi}(\lambda P t) & =\sum_{i=1}^{\infty} n_{i} \Phi\left(\lambda t_{i} u_{i}\right) \\
& =\sum_{i=1}^{j_{0}} n_{i} \Phi\left(\lambda t_{i} u_{i}\right)+\sum_{i=j_{0}+1}^{\infty} n_{i} \Phi\left(\lambda t_{i} u_{i}\right) \\
& \leq \sum_{i=1}^{j_{0}} n_{i} \Phi\left(\lambda t_{i} u_{i}\right)+\sum_{i=j_{0}+1}^{\infty} \frac{1}{2^{\frac{1}{p}+i}} \\
& <\sum_{i=1}^{j_{0}} n_{i} \Phi\left(\lambda t_{i} u_{i}\right)+1<\infty,
\end{aligned}
$$

which implies $P t \in l_{\Phi, p}$. Moreover, for any $t=\left(t_{1}, t_{2}, \ldots\right) \in c_{0}$, we have

$$
I_{\Phi}\left(\frac{P t}{\|t\|_{\infty}}\right)=\sum_{i=1}^{\infty} n_{i} \Phi\left(\frac{t_{i}}{\|t\|_{\infty}} u_{i}\right) \leq \sum_{i=1}^{\infty} \frac{1}{2^{\frac{1}{p}+i}}=2^{-\frac{1}{p}},
$$

then $\|P t\|_{\Phi, p} \leq 2^{\frac{1}{p}}\|P t\|_{\Phi, \infty} \leq\|t\|_{\infty}$.

On the other hand, for any $\lambda \in(0,1)$, there exists $j_{1} \in \mathbb{N}$ such that

$$
\frac{\left(1-\varepsilon_{j_{1}}\right)\left|t_{j_{1}}\right|}{\lambda \sup \left\{\left(1-\varepsilon_{i}\right)\left|t_{i}\right|: i \in \mathbb{N}\right\}}>1
$$

where $\varepsilon_{i}=\frac{1}{i+1}(i \in \mathbb{N})$, then

$$
\frac{\left|t_{j_{1}}\right|}{\lambda \sup \left\{\left(1-\varepsilon_{i}\right)\left|t_{i}\right|: i \in \mathbb{N}\right\}}>\frac{1}{1-\varepsilon_{j_{1}}}=1+\frac{1}{j_{1}}
$$

and

$$
I_{\Phi}\left(\frac{P t}{\lambda \sup \left\{\left(1-\varepsilon_{i}\right)\left|t_{i}\right|: i \in \mathbb{N}\right\}}\right)>n_{j_{1}} \Phi\left(\left(1+\frac{1}{j_{1}}\right) u_{j_{1}}\right)>2^{j_{1}+\frac{1}{p}+1} n_{j_{1}} \Phi\left(u_{j_{1}}\right)>1 .
$$


Therefore,

$$
\|P t\|_{\Phi, p} \geq\|P t\|_{\Phi, \infty} \geq \sup \left\{\left(1-\varepsilon_{i}\right)\left|t_{i}\right|: i \in \mathbb{N}\right\}
$$

which implies that $l_{\Phi, p}$ contains an asymptotically isometric copy of $c_{0}$.

Theorem 3.6 Let $1 \leq p \leq \infty, a_{\Phi}=0$, then $l_{\Phi, p}$ has the fixed point property if and only if it is reflexive.

Proof Since a reflexive Banach space $X$ with $\operatorname{WCS}(X)>1$ has the fixed point property, we only need to prove the necessity.

Suppose $\Phi \notin \Delta_{2}(0)$, then by Theorem $3.5, l_{\Phi, p}$ contains an asymptotically isometric copy of $c_{0}$. Hence $l_{\Phi, p}$ does not have the fixed point property.

Suppose $\Psi \notin \Delta_{2}(0)$, then there exists $y \in S\left(l_{\Psi, q}\right)$ such that $I_{\Psi}(l y)=\infty$ for any $l>1$, and for every sequence $\left\{\varepsilon_{n}\right\}$ decreasing to 0 , there exist $0=i_{1}<i_{2}<i_{3}<\cdots$ such that

$$
\left\|\sum_{i=i_{n}+1}^{i_{n+1}} y(i) e_{i}\right\|_{\Psi, q}>1-\varepsilon_{n} \quad \text { for all } n \in \mathbb{N} .
$$

Set $y_{n}=\sum_{i=i_{n}+1}^{i_{n+1}} y(i) e_{i}$, then there exists $x_{n} \in S\left(l_{\Phi, p}\right)$ such that $\left\langle y_{n}, x_{n}\right\rangle=\left\|y_{n}\right\|_{\Psi, q}$. Hence, for any $\alpha=(\alpha(n)) \in l^{1}$, we have

$$
\begin{aligned}
\sum_{n=1}^{\infty}|\alpha(n)| & =\sum_{n=1}^{\infty}|\alpha(n)|\left\|x_{n}\right\|_{\Phi, p} \geq\left\|\sum_{n=1}^{\infty} \alpha(n) x_{n}\right\|_{\Phi, p} \\
& \geq\left\langle\sum_{n=1}^{\infty} \alpha(n) x_{n}, \sum_{n=1}^{\infty} \operatorname{sign}(\alpha(n)) y_{n}\right\rangle \\
& =\sum_{n=1}^{\infty}|\alpha(n)|\left\langle x_{n}, y_{n}\right\rangle \geq\left(1-\varepsilon_{n}\right) \sum_{n=1}^{\infty}|\alpha(n)| .
\end{aligned}
$$

Hence $l_{\Phi, p}$ contains an asymptotically isometric copy of $l^{1}$. By Theorem 2 of [28], $l_{\Phi, p}$ does not have the fixed point property.

Theorem 3.7 If $a_{\Phi}>0,1 \leq p<\infty$, then $l_{\Phi, p}$ does not have the fixed point property.

Proof If $a_{\Phi}>0$, for any $n \in \mathbb{N}$, define $x_{n}=(\overbrace{0, \ldots, 0}^{(n-1)}, a_{\Phi}, 0, \ldots)$, then

$$
\begin{aligned}
& 1+I_{\Phi}\left(x_{n}\right)=1, \\
& \frac{1}{k}\left(1+I_{\Phi}^{p}\left(k x_{n}\right)\right)^{\frac{1}{p}}=\frac{1}{k}>1 \quad(\forall k \in(0,1)), \\
& \frac{1}{k}\left(1+I_{\Phi}^{p}\left(k x_{n}\right)\right)^{\frac{1}{p}}>1 \quad(\forall k>1) .
\end{aligned}
$$

Therefore, $\left\|x_{n}\right\|_{\Phi, p}=1$ for any $n \in \mathbb{N}$.

Moreover, we can prove that $\left\|\sum_{n=1}^{\infty} x_{n}\right\|_{\Phi, p}=1$. Hence, define $P: l^{\infty} \rightarrow l_{\Phi, p}$ by

$$
P y=\sum_{n=1}^{\infty} y_{n} x_{n}
$$


for all $y=\left(y_{1}, \ldots, y_{n}, \ldots\right) \in l^{\infty}$. Then the operator $P$ is obviously linear, since

$$
\begin{aligned}
\|y\|_{\infty} & =\sup _{n}\left|y_{n}\right|=\sup _{n}\left|y_{n}\right|\left\|x_{n}\right\|_{\Phi, p} \leq\left\|\sum_{n=1}^{\infty} y_{n} x_{n}\right\|_{\Phi, p} \\
& =\|P y\|_{\Phi, p} \leq \sup _{n}\left|y_{n}\right|\left\|\sum_{n=1}^{\infty} x_{n}\right\|_{\Phi, p}=\sup _{n}\left|y_{n}\right|=\|y\|_{\infty},
\end{aligned}
$$

so we have $\|P y\|_{\Phi, p}=\|y\|_{\infty}$, then $P$ is an order isometry of $l^{\infty}$ onto a closed subspace $P\left(l^{\infty}\right)$ of $l_{\Phi, p}$.

\section{Competing interests}

The authors declare that they have no competing interests.

\section{Authors' contributions}

All authors contributed equally and significantly in writing this paper. All authors read and approved the final manuscript.

\section{Author details}

${ }^{1}$ Department of Mathematics, Harbin Institute of Technology, Harbin, 150001, P.R. China. ${ }^{2}$ Department of Mathematics, Harbin Normal University, Harbin, 150025, P.R. China. ${ }^{3}$ Department of Mathematics, Harbin University of Science and Technology, Harbin, 150080, P.R. China. ${ }^{4}$ Faculty of Mathematics and Computer Science, Adam Mickiewicz University, ul. Umultowska 87, Poznań, 61-614, Poland.

\section{Acknowledgements}

This work was supported by the Provincial Education Department Fund (12531185) and partly supported by the National Natural Science Foundation of China (61203191)

Received: 12 August 2013 Accepted: 20 November 2013 Published: 13 Dec 2013

\section{References}

1. Opial, Z: Weak convergence of the sequence of successive approximations of nonexpansive mappings. Bull. Am. Math. Soc. 73, 591-597 (1967)

2. Prus, S: Banach spaces with the uniform Opial property. Nonlinear Anal. 18, 697-704 (1992)

3. Lin, PK, Tan, KK, Xu, HK: Demiclosedness principle and asymptotic behavior for asymptotically nonexpansive mappings. Nonlinear Anal. 24, 929-946 (1995)

4. Ayerbe, JM, Benavides, TD, Acedo, GL: Measures of Noncompactness in Metric Fixed Point Theory. Birkhäuser, Basel (1997)

5. Kirk, WA, Sims, B (eds.): Handbook of Metric Fixed Point Theory. Kluwer Academic, Dordrecht (2001)

6. Krasnoselskii, MA, Rutickii, YB: Convex Functions and Orlicz Spaces. Noordhoff, Groningen (1961)

7. Goebel, K, Kirk, WA: Topics in Metric Fixed Point Theory. Cambridge University Press, Cambridge (1990)

8. Goebel, K, Reich, S: Uniform Convexity, Hyperbolic Geometry and Nonexpansive Mappings. Dekker, New York (1984)

9. Gornicki, J: Some remarks on almost convergence of the Picard iterates for nonexpansive mappings in Banach spaces which satisfy the Opial condition. Comment. Math. 29, 59-68 (1988)

10. Bynum, WL: Normal structure coefficients for Banach spaces. Pac. J. Math. 86, 427-436 (1980)

11. Sims, B, Smyth, A: On some Banach space properties sufficient for weak normal structure and their permanence properties. Trans. Am. Math. Soc. 351(2), 497-513 (1999)

12. Cui, YA, Duan, LF, Hudzik, H, Wisla, M: Basic theory of $p$-Amemiya norm in Orlicz spaces $(1 \leq p \leq \infty)$ : extreme points and rotundity in Orlicz spaces endowed with these norms. Nonlinear Anal. 69, 1797-1816 (2008)

13. Cui, YA, Hudzik, H, Li, JJ, Wisla, M: Strongly extreme points in Orlicz spaces equipped with the $p$-Amemiya norm. Nonlinear Anal. 71, 6343-6364 (2009)

14. Cui, YA, Hudzik, H, Wisla, M, Wlazlak, K: Non-squareness properties of Orlicz spaces equipped with the $p$-Amemiya norm. Nonlinear Anal. 75, 3973-3993 (2012)

15. Chen, LL, Cui, YA: Complex extreme points and complex rotundity in Orlicz function spaces equipped with the p-Amemiya norm. Nonlinear Anal. 73, 1389-1393 (2010)

16. Chen, LL, Cui, YA: Complex rotundity of Orlicz sequence spaces equipped with the $p$-Amemiya norm. J. Math. Anal. Appl. 378, 151-158 (2011)

17. Chen, ST: Geometry of Orlicz Spaces. Dissertationes Mathematicae, vol. 356. Istitute of Mathematics, Warszawa (1996)

18. Maligranda, L: Orlicz Spaces and Interpolation. Seminars in Math., vol. 5. Universidade Estadual de Campinas, Campinas (1989)

19. Musielak, J: Orlicz Spaces and Modular Spaces. Lecture Notes in Math., vol. 1034. Springer, Berlin (1983)

20. Li, XY, Cui, YA: The dual space of Orlicz space equipped with p-Amemiya norm. J. Harbin Univ. Sci. Technol. 16(1), $110-112(2011)$

21. Cui, YA: Weakly convergent sequence coefficient in Köthe sequence spaces. Proc. Am. Math. Soc. 126, 195-201 (1998)

22. Cui, YA, Hudzik, H: On the uniform Opial property in some modular sequence spaces. Funct. Approx. Comment. Math. 26, 93-102 (1998) 
23. Cui, YA, Hudzik, H, Zhu, HW: Maluta's coefficient of Musielak-Orlicz sequence spaces equipped with Orlicz norm. Proc Am. Math. Soc. 126, 115-121 (1998)

24. Cui, YA, Hudzik, H: Maluta's coefficient and Opial's properties in Musielak-Orlicz sequence spaces equipped with the Luxemburg norm. Nonlinear Anal. 35, 475-485 (1999)

25. Cui, YA, Hudzik, H, Yu, FF: On Opial properties and Opial modulus for Orlicz sequence spaces. Nonlinear Anal. 55, 335-350 (2003)

26. Yao, H, Wang, T: Maluta's coefficient of Musielak-Orlicz sequence spaces. Acta Math. Sin. Engl. Ser. 21, 699-704 (2005)

27. Zhang, GL: Weakly convergent sequence coefficient of product space. Proc. Am. Math. Soc. 117(3), 637-643 (1992)

28. Dowling, PN, Lennai, CJ, Turett, B: Reflexivity and fixed-point property for nonexpansive maps. J. Math. Anal. Appl. 200, 653-662 (1996)

10.1186/1687-1812-2013-340

Cite this article as: He et al.: The fixed point property of Orlicz sequence spaces equipped with the $p$-Amemiya norm. Fixed Point Theory and Applications 2013, 2013:340

Submit your manuscript to a SpringerOpen ${ }^{\circ}$ journal and benefit from:

- Convenient online submission

- Rigorous peer review

- Immediate publication on acceptance

Open access: articles freely available online

- High visibility within the field

- Retaining the copyright to your article

Submit your next manuscript at $>$ springeropen.com 